\title{
Selective and stoichiometric incorporation of ATP by self- assembling amyloid fibrils.
}

Robert Dec, ${ }^{1}$ Wojciech Puławski, ${ }^{2}$ and Wojciech Dzwolak ${ }^{*}$

${ }^{1}$ Faculty of Chemistry, Biological and Chemical Research Centre, University of Warsaw, 1 Pasteur Street, 02-093 Warsaw, Poland. 'Institute of High Pressure Physics, Polish Academy of Sciences, 29/37 Sokołowska Street, 01-142 Warsaw, Poland

* Corresponding author: Dzwolak; Phone: +48 22552 6567; Fax: +48 225524029 ; E-mail:

wdzwolak@,chem.uw.edu.pl 


\begin{abstract}
ATP acts as a biological hydrotrope preventing protein aggregation. Here, we report a novel chimeric peptide, $\mathrm{ACC}_{1-13} \mathrm{~K}_{8}$, with an unusual capacity to bind and incorporate ATP while self-assembling into amyloid fibrils. The amino acid sequence combines highly amyloidogenic segment of insulin's A-chain $\left(\mathrm{ACC}_{1-13}\right)$ and octalysine $\left(\mathrm{K}_{8}\right)$. Fibrillization requires binding 2 ATP molecules per $\mathrm{ACC}_{1-13} \mathrm{~K}_{8}$ monomer and is not triggered by adenosine di- and monophosphates (ADP, AMP). Infrared and CD spectra and AFM-based morphological analysis reveal tight and orderly entrapment of ATP within superstructural hybrid peptide-ATP fibrils. The incorporation of ATP is an emergent property of $\mathrm{ACC}_{1-13} \mathrm{~K}_{8}$ not observed for $\mathrm{ACC}_{1-13}$ and $\mathrm{K}_{8}$ segments separately. We demonstrate how new functionalities (e.g. ATP storage) emerge from synergistic coupling of amyloidogenic segments with non-amyloidogenic peptide ligands, and suggest that ATP's role in protein misfolding is more nuanced than previously assumed.
\end{abstract}




\section{INTRODUCTION}

Apart from its canonical function as 'the energy currency of the cell', ATP has recently been recognized as an important hydrotrope preventing formation of protein aggregates. ${ }^{1,2}$ Those include amyloid fibrils, the highly ordered $\beta$-sheet-rich entities whose presence in vivo has been linked to various disorders e.g. Alzheimer's disease, ${ }^{3,4}$ as well as benign biological functions such as hormone storage. ${ }^{5,6}$ The idea that amyloidogenic condensation of simple peptides played a role in the prebiotic world (the pre-RNA era) has also been raised. ${ }^{7,8}$ Upon binding of ATP, local charge distribution and solvation of protein surfaces may change significantly. This, in turn, explains the observed impact of ATP on proteinaceous liquid droplets formed through liquid-liquid phase separation (LLPS), ${ }^{1,9,10.11}$ the ubiquitous phenomenon ${ }^{12}$ intimately involved both in proper cellular functions, ${ }^{13,14}$ and in disease. ${ }^{15,16,17}$ Elsewhere, the concept of LLPS inspired an intellectually compelling idea of how compartmentalization could have been achieved in early membraneless protocells. ${ }^{18,19,20,21,22}$ Although many amyloidogenic proteins are also prone to LLPS $^{23,24,25,26,27}$ a detailed mechanistic description of the relationship between transition pathways leading to liquid droplets and amyloid fibrils remains elusive. ${ }^{28}$ Koga et al. have shown that at a close-to-neutral $\mathrm{pH}$ the electrostatics-driven binding of ATP and polylysine triggers LLPS. ${ }^{29}$ This finding has made the polylysine-ATP pair an attractive model for studies of liquid droplets also in the prebiotic context. ${ }^{30,31}$ Except for a strongly alkaline environment, ${ }^{32,33}$ polylysine is not amyloidogenic and this has sparked our initial curiosity as to whether tethering an amyloidogenic peptide fragment to an oligo-lysine segment could switch the ATP-induced transition pathway from LLPS to one leading to amyloid fibrils. This has led us to report here for the first time an amyloid system in which stoichiometric incorporation of ATP is the necessary condition of the fibrillar self-assembly.

\section{RESULTS AND DISCUSSION}


The disulfide-constrained N-terminal fragment of insulin's A-chain encompassing the first 13 residues $\left(\mathrm{ACC}_{1-13}\right)$ is in the $\alpha$-helical conformation while remaining an integral part of the intact hormone molecule

(Fig. 1a) but reveals its extremely amyloidogenic character when separated from it. ${ }^{34,35}$
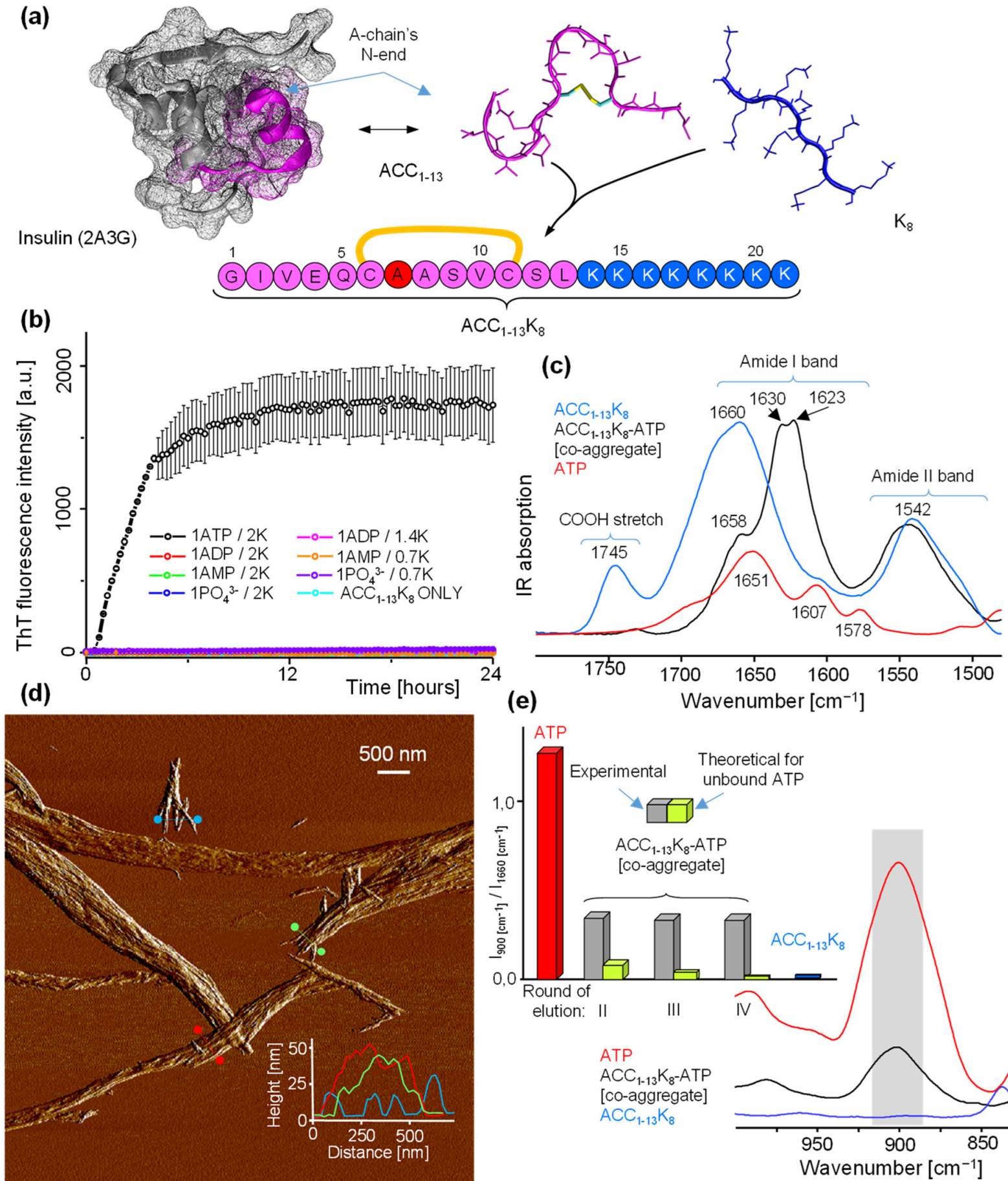

(e)

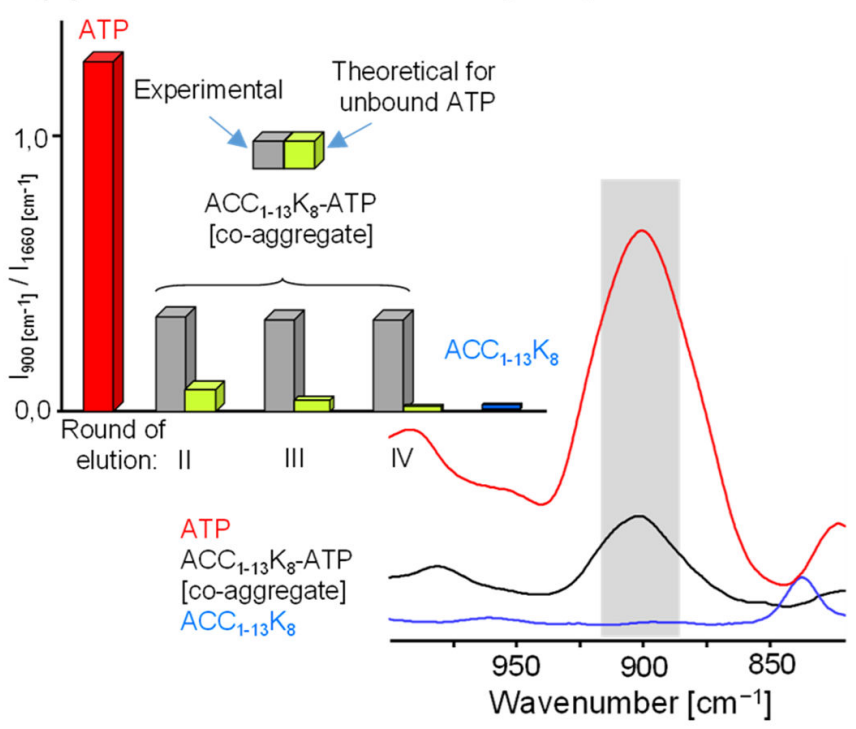


Figure 1. Design and ATP-dependent fibrillization of $\mathrm{ACC}_{1-13} \mathrm{~K}_{8}$ peptide. (a) The $\mathrm{ACC}_{1-13} \mathrm{~K}_{8}$ amino acid sequence consisting of the first $13 \mathrm{~N}$-terminal residues of bovine insulin's A-chain (PDB entry: 2A3G) extended at the C-end by additional 8 lysine residues. (b) De novo fibrillization kinetics of ACC $_{1-}$ ${ }_{13} \mathrm{~K}_{8}\left(0.15 \mathrm{mM}, \mathrm{pH} 6.5,37^{\circ} \mathrm{C}\right)$ probed by ThT fluorescence assay in the presence of ATP, ADP, AMP and sodium phosphate at the indicated molar ratios to lysine residues. (c) Infrared spectrum of $\mathrm{ACC}_{1-13} \mathrm{~K}_{8-}$ ATP co-aggregate collected afterward overlaid with spectra of non-aggregated $\mathrm{ACC}_{1-13} \mathrm{~K}_{8}$ and ATP. (d) AFM amplitude image of $\mathrm{ACC}_{1-13} \mathrm{~K}_{8}$-ATP aggregate. Superimposed are cross-sections of selected specimen. (e) Impact of repetitive washing of $\mathrm{ACC}_{1-13} \mathrm{~K}_{8}$-ATP amyloid with water on the relative intensity of the $900 \mathrm{~cm}^{-1}$ ATP infrared band.

When attached to certain moderately long non-amyloidogenic peptide chains $\mathrm{ACC}_{1-13}$ enforces cooperative amyloidogenic self-assembly and adoption of the common parallel $\beta$-sheet structure. ${ }^{36}$

In this study, the amino acid sequence of $\mathrm{ACC}_{1-13}$ was extended at its $\mathrm{C}$-end by an octalysine $\left(\mathrm{K}_{8}\right)$ segment (Fig. 1a); the $\mathrm{ACC}_{1-13} \mathrm{~K}_{8}$ peptide was obtained through solid phase synthesis (Supporting Information - section $\mathrm{A})$. At neutral $\mathrm{pH}$, an $\mathrm{ACC}_{1-13} \mathrm{~K}_{8}$ monomer has a net electric charge of +7 ( +8 on the $\mathrm{K}_{8}$ part and -1 on residue E4) which, in the absence of charge-compensating anions, rules out aggregation. Thioflavin $\mathrm{T}$ (ThT) fluorescence-based kinetic assay was employed to examine $\mathrm{ACC}_{1-13} \mathrm{~K}_{8}$ fibrillization in the presence of ATP, ADP, AMP and sodium phosphate at excess concentrations sufficient to compensate, upon binding, for the peptide's electric charge (Fig. 1b), see Supporting Information sections $\mathrm{B}$ and $\mathrm{C}$ for details. Only when ATP was added, a pronounced increase in ThT emission intensity was observed suggesting that formation of possibly amyloid-like aggregate is taking place. The process was fast with negligible lag phase and was complete within 10 hours of incubation at $37^{\circ} \mathrm{C}$. According to the IR spectra of precipitates collected at the end of the kinetic experiment (Fig. 1c) ATP-aggregated ACC 1 ${ }_{13} \mathrm{~K}_{8}$ acquires parallel $\beta$-sheet structure: the amid I band's contour, frequency, and bandwidth are very similar to those of insulin amyloid fibrils. ${ }^{36}$ The splitting of the main spectral component (here at 1623 $\left.\mathrm{cm}^{-1} / 1630 \mathrm{~cm}^{-1}\right)$ is also found in infrared spectra insulin fibrils. Fibrillar nature of the aggregate was confirmed with atomic force microscopy (AFM), see Fig. 1d. Interestingly, single fibrillar specimen were rare in samples left to co-aggregate for 24 hours. Instead, tightly packed superstructural arrangements appear to dominate morphological landscape of mature $\mathrm{ACC}_{1-13} \mathrm{~K}_{8}$-ATP. Such morphological forms are 
reminiscent of the chiral insulin amyloid superstructures with strong chiroptical properties reported earlier. ${ }^{37,38}$ Some of the tiniest fibrils shown in Fig. $1 \mathrm{~d}$ are $10 \mathrm{~nm}$ in diameter implying presence of intertwined protofilaments. Thus we have carried out an additional time-lapse AFM analysis of gradually forming $\mathrm{ACC}_{1-13} \mathrm{~K}_{8}$-ATP fibrils (Supporting Information - section D) which revealed sporadic short fibrils approximately $4-5 \mathrm{~nm}$ in diameter present already after 10 minutes of incubation. This type of morphology dominates the earliest stages of fibrillization, however samples collected after 2 hours of incubation contain already the thicker superstructures which co-exist with relatively flat assemblies of tightly aligned fibrils. These various amyloid forms share similarities with the aforementioned insulin amyloid superstructures. ${ }^{37,38}$ Subsequently, we have taken advantage of the fact that some of the vibrational bands of ATP are not overlapped with the bands arising from the peptide backbone in order to confirm that the precipitated $\mathrm{ACC}_{1-13} \mathrm{~K}_{8}$ amyloid does contain the nucleotide. We probed relative intensity of the band at ca. $900 \mathrm{~cm}^{-1}$ stemming from adenosine moiety scaled to the IR absorption intensity at $1660 \mathrm{~cm}^{-1}$ (Fig. 1e). Clearly, in contrast to sole $\mathrm{ACC}_{1-13} \mathrm{~K}_{8}$ peptide, $\mathrm{ACC}_{1-13} \mathrm{~K}_{8}$-ATP amyloid exhibits pronounced and persistent absorption at this frequency which is not affected by the following rounds of elution with water and centrifuging. This, in turn, not only provides direct evidence that ATP is incorporated within the ACC $_{1-}$ ${ }_{13} \mathrm{~K}_{8}$ amyloid, but also enables a straightforward verification of the molar peptide : nucleotide coaggregation ratio by UV-spectrophotometric measurements of the remaining ATP concentration in the supernatant over co-aggregate. Arguably, the determined 1:2 ratio is intuitive given that at neutral pH ATP exists predominantly in the form of $\mathrm{ATP}^{4-}$ anion with only a small fraction of $\mathrm{ATP}^{3-} \cdot{ }^{39}$

The strict stoichiometry of the $\mathrm{ACC}_{1-13} \mathrm{~K}_{8}$-ATP co-aggregation is also reflected in the titration experiment reported in Fig. 2a. When the peptide is practically saturated with ATP (number of lysine residues per ATP is 4:1 or less) the kinetic trajectories of ThT-fluorescence-monitored fibrillization are similar and the final emission plateaus reach similar values reflecting comparable amounts of formed amyloid. However, with the ratio increasing to $6: 1$ and beyond the fibrillization abruptly decelerates and becomes ineffective. This observation is not surprising given that an $\mathrm{ACC}_{1-13} \mathrm{~K}_{8}$ monomer binding a single 
ATP molecule retains relatively large $(+3)$ net charge which prevents aggregation. On the other hand, the fraction of peptide monomers binding 2 ATP units would be negligible due to both entropic and enthalpic factors (the electrostatics favoring ATP binding to free peptide monomers).

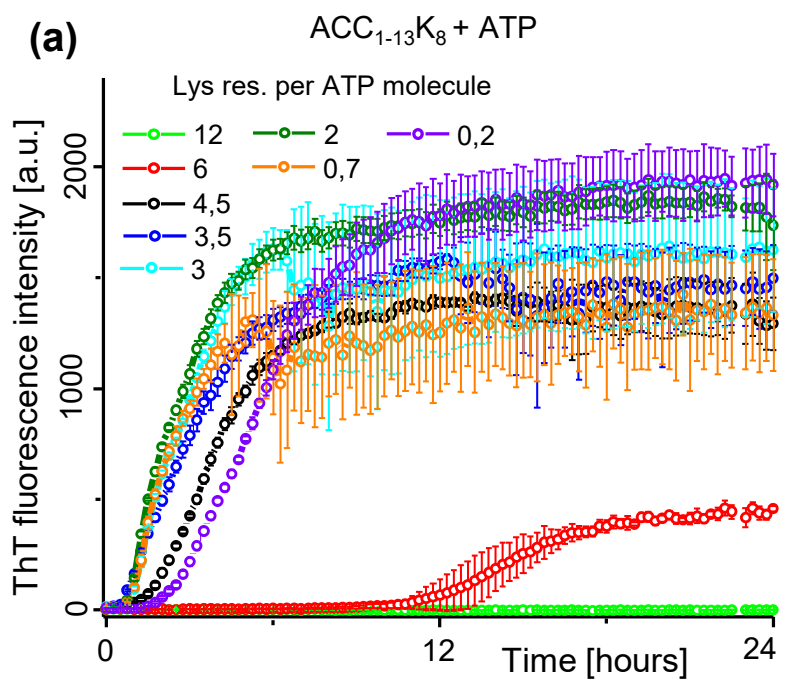

(b)

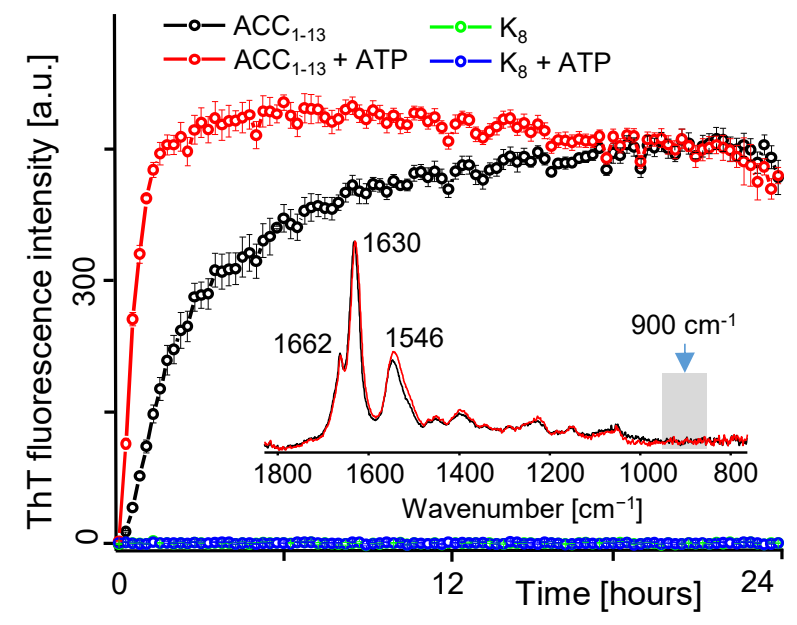

Figure 2. De novo fibrillization kinetics of $\mathrm{ACC}_{1-13} \mathrm{~K}_{8}$ (a) and the constituent $\mathrm{ACC}_{1-13}$ and $\mathrm{K}_{8}$ peptides (b) in the presence of ATP. All peptides were dissolved at $0.15 \mathrm{mM}$ concentration, $\mathrm{pH} 6.5$, lysine res. : ATP molar ratios are indicated. In experiments with $\mathrm{ACC}_{1-13}$ and $\mathrm{K}_{8}$, the ATP conc. was $0.45 \mathrm{mM}$. Inset in panel (b) shows infrared spectra of $\mathrm{ACC}_{1-13}$ fibrils formed in the presence and absence of ATP and subsequently washed with 5-fold excess of water. 
The control experiment summarized in Fig. $2 b$ highlights the crucial synergistic action of both $\mathrm{ACC}_{1-13}$ and $\mathrm{K}_{8}$ segments. On its own, free $\mathrm{K}_{8}$ peptide does not form fibrils in the presence of ATP while the profoundly amyloidogenic $\mathrm{ACC}_{1-13}$ does fibrillate regardless of ATP presence. Remarkably, ATP appears to accelerate $\mathrm{ACC}_{1-13}$ aggregation, however, the IR data collected afterwards shows no evidence of ATP entrapment (inset in Fig. 2b). It was recently reported that fibrillization of tau protein (fragment $\mathrm{K} 18$ ) is accelerated in the presence of ATP, however the nucleotide is not bound to the aggregate. ${ }^{40}$

In the case of hierarchically intertwined insulin amyloid whose morphology is similar to the one reported here for the $\mathrm{ACC}_{1-13} \mathrm{~K}_{8}$-ATP aggregate, the superstructural chirality manifests itself also in strong chiroptical properties. ${ }^{41}$ Specifically, electronic transitions in amyloid-bound dyes such as ThT or Congo red become active in corresponding circular dichroism (CD) spectra as a result of coupling of transition moments facilitated by the orderly binding to the amyloid surface. ${ }^{37,38}$ Hence, we sought to determine whether a similar phenomenon involving electronic transitions in adenine moieties occurs upon formation of $\mathrm{ACC}_{1-13} \mathrm{~K}_{8}$-ATP amyloid. Of the several CD-active electronic transitions in an unbound ATP monomer (Fig 3a, right inset), only the one at $256 \mathrm{~nm}$ lies outside of the wavelength range overlapped with $\pi-\pi^{*}$ and $\mathrm{n}-\pi^{*}$ electronic transitions of $\mathrm{ACC}_{1-13} \mathrm{~K}_{8}$ amide chromophores.

The time-lapse CD spectra in Fig. 3a correspond to the progressing co-aggregation of $\mathrm{ACC}_{1-13} \mathrm{~K}_{8}$ and ATP. While interpretation of the spectral changes taking place in the short $(<250 \mathrm{~nm})$ wavelength range is complicated by the superposition of amide and adenine chromophore signals, analysis of the unobscured band at $256 \mathrm{~nm}$ is more straightforward. There are three striking spectral changes in this region taking place upon aggregation: [i] reversal of the CD sign, [ii] bathochromic shift to approximately $278 \mathrm{~nm}$, and [iii] pronounced increase in the CD intensity (estimated as 25 times versus unbound ATP at $256 \mathrm{~nm})$. These observations imply that the fibrillization process facilitates highly ordered chiral arrangements of adenine moieties and, in turn, coupling of electronic transition moments required for the induced CD signals to emerge. 
That a tight alignment of adenine moieties into stacks is structurally compatible with the saturation of coulombic interactions between triphosphates and charged lysine side chains required for the stabilization of amyloid is, in fact, supported by molecular dynamics (MD) simulations of ATP docking onto surface of pre-assembled $\mathrm{ACC}_{1-13} \mathrm{~K}_{8}$ fibril (see Fig. 3b, and Supporting Information sections E and F).

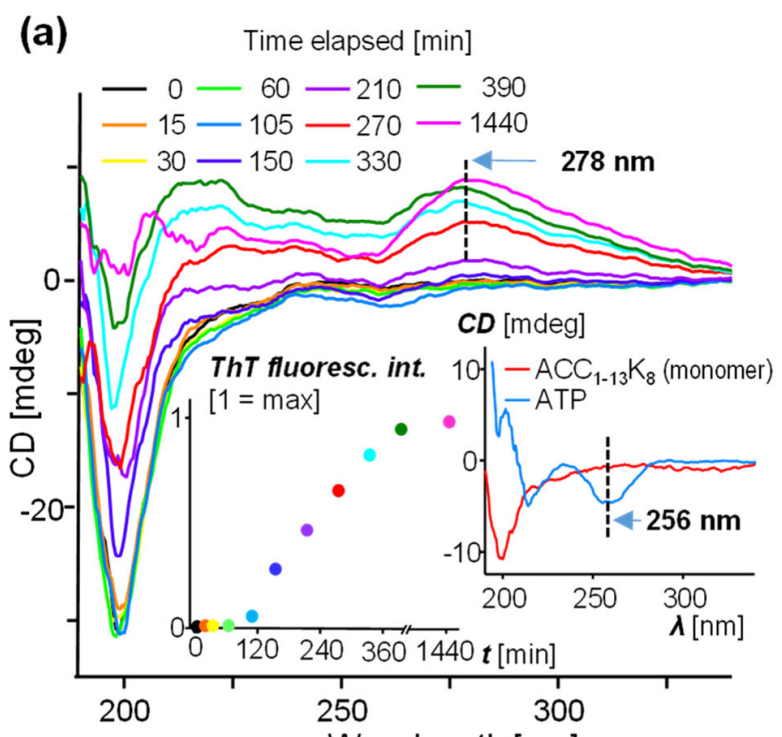

(b)

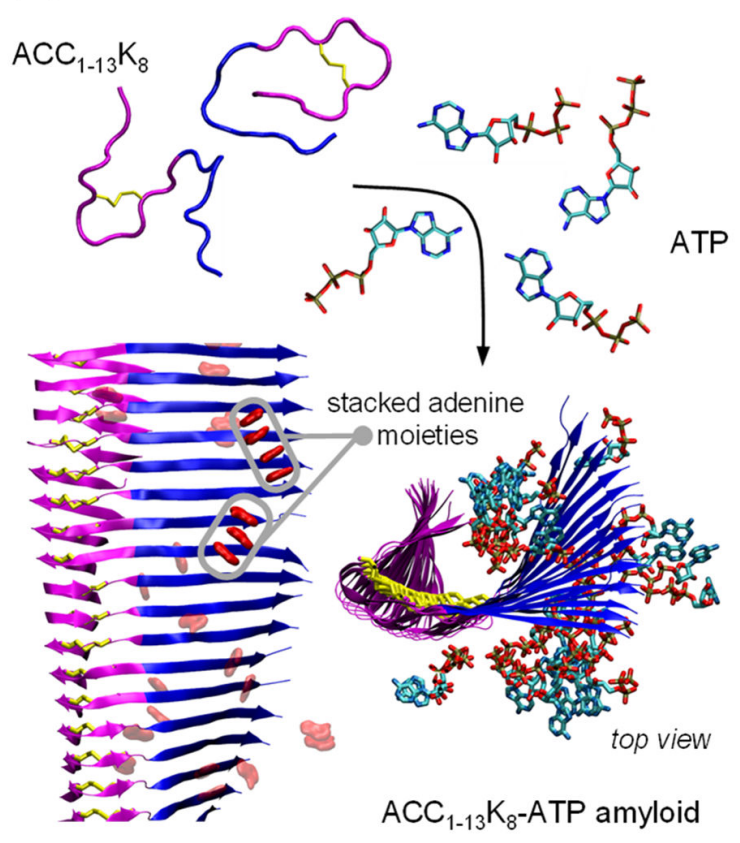


Figure 3. Ordering of adenine moieties within $\mathrm{ACC}_{1-13} \mathrm{~K}_{8}$-ATP amyloid. (a) Time-lapse induced CD spectra of gradually co-aggregating $\mathrm{ACC}_{1-13} \mathrm{~K}_{8}(0.15 \mathrm{mM})$ and $\mathrm{ATP}(0.45 \mathrm{mM})$ at $\mathrm{pH} 6.5$ and $37^{\circ} \mathrm{C}$. The corresponding ThT emission intensity is indicated in the left inset. CD spectra of free monomeric $\mathrm{ACC}_{1-}$ ${ }_{13} \mathrm{~K}_{8}$ and ATP are shown in the right inset. (b) Schematic visualization of the co-aggregation process leading to the hybrid peptide-ATP amyloid fibrils. The oligolysine segments are decorated with stacked adenine moieties of incorporated ATP molecules.

Although oligolysine peptides undergo the LLPS transition upon ATP binding we have not observed liquid droplets in fresh or aged mixtures of $\mathrm{ACC}_{1-13} \mathrm{~K}_{8}$ with either ATP, ADP or AMP. The fact that linking of oligolysine to a potent amyloidogenic segment changes the thermodynamically plausible structural phases and accessible transition pathways is not surprising. However, the observation that the hybrid $\mathrm{ACC}_{1-13} \mathrm{~K}_{8}$ peptide selectively and stoichiometrically binds and incorporates ATP within the growing amyloid fibril is significant. Based on this example, a versatile and intuitive design strategy (merger of target-recognizing and amyloidogenic segments) toward fibrillar molecular carriers for small charged molecules may be put forward. As has been demonstrated in this study, the binding strength in such systems is amplified by repetitiveness and cooperativity afforded by the amyloidogenic self-assembly. ${ }^{4}$ In the case of $\mathrm{ACC}_{1-13} \mathrm{~K}_{8}$, the entrapment of ATP molecules emerges through the synergy of the properties of both peptide segments. More importantly, this work highlights the possibility that the role ATP plays in disease-associated misfolding of certain proteins may be more nuanced. RNA is recognized to bind and trigger amyloidogenesis of proteins such as PrPC ${ }^{42}$ and TDP-43. ${ }^{43}$ However, this capacity appears not to be restricted to oligonucleotides. The physiological concentrations of ATP are relatively high ${ }^{9}$ and may exceed by an order of magnitude those used to trigger $\mathrm{ACC}_{1-13} \mathrm{~K}_{8}$ aggregation in our study. Hence ATP could exercise influence analogous to RNA on certain proteins in which lysine-rich (or perhaps more generally: basic) amino acid segments are in proximity to amyloidogenic fragments.

MATERIALS AND METHODS: see the Supporting Information 


\section{Supporting Information}

Detailed experimental procedures, additional time-lapse AFM data and in silico modelling of ATP binding within $\mathrm{ACC}_{1-13} \mathrm{~K}_{8}-\mathrm{ATP}$ complex amyloid.

\section{ACKNOWLEDGMENT}

This work was supported by the National Science Centre of Poland, grant no. 2017/25/B/ST5/02599.

\section{Conflict of interest}

The authors declare no conflict of interest.

Keywords: · aggregation $\cdot$ amyloid $\cdot$ ATP $\cdot$ fibrils $\cdot$ self-assembly

\section{REFERENCES}

(1) Patel, A.; Malinovska, L.; Saha, S.; Wang, J.; Alberti, S.; Krishnan, Y.; Hyman, A. A. Science 2017, 356 (6339), 753-756.

(2) Rice, A. M.; Rosen, M. K. Science 2017, 356 (6339), 701-702.

(3) Chiti, F.; Dobson, C. M. Annu. Rev. Biochem. 2017, 86, 27-68.

(4) Riek, R.; Eisenberg, D. S. Nature 2016, 539 (7628), 227-235.

(5) Fowler, D. M.; Koulov, A. V.; Balch, W. E.; Kelly, J. W. Trends Biochem. Sci. 2007, 32 (5), $217-$ 224.

(6) Maji, S. K.; Perrin, M. H.; Sawaya, M. R.; Jessberger, S.; Vadodaria, K.; Rissman, R. A.; Singru, P. S.; Nilsson, K. P. R.; Simon, R.; Schubert, D.; Eisenberg, D.; Rivier, J.; Sawchenko, P.; Vale, W.; Riek, R. Science 2009, 325 (5938), 328-332.

(7) Greenwald, J.; Friedmann, M. P.; Riek, R. Angew. Chem. 2016, 128 (38), 11781-11785.

(8) Maury, C. P. J. Amyloid and the Origin of Life: Cell. Mol. Life Sci. 2018, 75 (9), 1499-1507.

(9) Kang, J.; Lim, L.; Song, J. Commun. Biol. 2019, 2, 223.

(10) Kang, J.; Lim, L.; Song, J. Biochem. Biophys. Res. Commun. 2018, 504 (2), 545-551.

(11) Hayes, M. H.; Peuchen, E. H.; Dovichi, N. J.; Weeks, D. L. eLife 2018, 7, e35224.

(12) Hyman, A. A.; Weber, C. A.; Jülicher, F. Annu. Rev. Cell Dev. Biol. 2014, 30, 39-58.

(13) Brangwynne, C. P.; Eckmann, C. R.; Courson, D. S.; Rybarska, A.; Hoege, C.; Gharakhani, J.; Jülicher, F.; Hyman, A. A. Science 2009, 324 (5935), 1729-1732.

(14) Larson, A. G.; Elnatan, D.; Keenen, M. M.; Trnka, M. J.; Johnston, J. B.; Burlingame, A. L.; Agard, D. A.; Redding, S.; Narlikar, G. J. Nature 2017, 547 (7662), 236-240.

(15) Alberti, S.; Dormann, D. Annu. Rev. Genet. 2019, 53, 171-194.

(16) Zbinden, A.; Pérez-Berlanga, M.; De Rossi, P.; Polymenidou, M. Dev. Cell 2020, 55 (1), 45-68.

(17) Shin, Y.; Brangwynne, C. P. Science 2017, 357 (6357).

(18) Oparin, A. I., The Origin of Life, MacMillian Co., New York, (1938). 
(19) Drobot, B.; Iglesias-Artola, J. M.; Le Vay, K.; Mayr, V.; Kar, M.; Kreysing, M.; Mutschler, H.; Tang, T.-Y. D. Nat. Commun. 2018, 9 (1), 3643.

(20) Crowe, C. D.; Keating, C. D. Interface Focus 2018, 8 (5), 20180032.

(21) Alshareedah, I.; Moosa, M. M.; Raju, M.; Potoyan, D. A.; Banerjee, P. R. Proc. Natl. Acad. Sci. U. S. A. 2020, 117 (27), 15650-15658.

(22) Cinar, H.; Fetahaj, Z.; Cinar, S.; Vernon, R. M.; Chan, H. S.; Winter, R. H. A. Chem.-Eur. J. 2019, 25 (57), 13049-13069.

(23) de Oliveira, G. A. P.; Cordeiro, Y.; Silva, J. L.; Vieira, T. C. R. G. Adv. Protein Chem. Struct. Biol. 2019, 118, 289-331.

(24) Ray, S.; Singh, N.; Kumar, R.; Patel, K.; Pandey, S.; Datta, D.; Mahato, J.; Panigrahi, R.; Navalkar, A.; Mehra, S.; Gadhe, L.; Chatterjee, D.; Sawner, A. S.; Maiti, S.; Bhatia, S.; Gerez, J. A.; Chowdhury, A.; Kumar, A.; Padinhateeri, R.; Riek, R.; Krishnamoorthy, G.; Maji, S. K. Nat. Chem. 2020, 12 (8), 705716.

(25) Babinchak, W. M.; Surewicz, W. K. J. Mol. Biol. 2020, 432 (7), 1910-1925.

(26) Tange, H.; Ishibashi, D.; Nakagaki, T.; Taguchi, Y.; Kamatari, Y. O.; Ozawa, H.; Nishida, N. J. Biol. Chem. 2021, 100367.

(27) Cinar, S.; Cinar, H.; Chan, H. S.; Winter, R. J. Am. Chem. Soc. 2019, 141 (18), 7347-7354.

(28) Xing, Y.; Nandakumar, A.; Kakinen, A.; Sun, Y.; Davis, T. P.; Ke, P. C.; Ding, F. J. Phys. Chem. Lett. 2021, 12 (1), 368-378.

(29) Koga, S.; Williams, D. S.; Perriman, A. W.; Mann, S. Nat. Chem. 2011, 3 (9), 720-724.

(30) Mann, S. Angew. Chem. Int. Ed Engl. 2013, 52 (1), 155-162.

(31) Fetahaj, Z.; Ostermeier, L.; Cinar, H.; Oliva, R.; Winter, R. J. Am. Chem. Soc. 2021, 143 (13), $5247-5259$.

(32) Fändrich, M.; Dobson, C. M. EMBO J. 2002, 21 (21), 5682-5690.

(33) Dzwolak, W.; Ravindra, R.; Nicolini, C.; Jansen, R.; Winter, R. J. Am. Chem. Soc. 2004, 126 (12), 3762-3768.

(34) Piejko, M.; Dec, R.; Babenko, V.; Hoang, A.; Szewczyk, M.; Mak, P.; Dzwolak, W. J. Biol. Chem. 2015, 290 (10), 5947-5958.

(35) Dec, R.; Koliński, M.; Dzwolak, W. FEBS J. 2019, 286 (16), 3194-3205.

(36) Dec, R.; Dzwolak, W. Langmuir 2020, 36 (41), 12150-12159.

(37) Dzwolak, W.; Loksztejn, A.; Galinska-Rakoczy, A.; Adachi, R.; Goto, Y.; Rupnicki, L. J. Am. Chem. Soc. 2007, 129 (24), 7517-7522.

(38) Loksztejn, A.; Dzwolak, W. J. Mol. Biol. 2010, 395 (3), 643-655.

(39) Storer, A. C.; Cornish-Bowden, A. Biochem. J. 1976, 159 (1), 1-5.

(40) Heo, C. E.; Han, J. Y.; Lim, S.; Lee, J.; Im, D.; Lee, M. J.; Kim, Y. K.; Kim, H. I. ACS Chem. Neurosci. 2020, 11 (19), 3144-3152.

(41) Dzwolak, W. Chirality 2014, 26 (9), 580-587.

(42) Geoghegan, J. C.; Valdes, P. A.; Orem, N. R.; Deleault, N. R.; Williamson, R. A.; Harris, B. T.; Supattapone, S. J. Biol. Chem. 2007, 282 (50), 36341-36353.

(43) Vogler, T. O.; Wheeler, J. R.; Nguyen, E. D.; Hughes, M. P.; Britson, K. A.; Lester, E.; Rao, B.; Betta, N. D.; Whitney, O. N.; Ewachiw, T. E.; Gomes, E.; Shorter, J.; Lloyd, T. E.; Eisenberg, D. S.; Taylor, J. P.; Johnson, A. M.; Olwin, B. B.; Parker, R. Nature 2018, 563 (7732), 508-513. 


\section{Table of Contents graphical abstract}

The merger of insulin's amyloidogenic segment and an octalysine stretch produces a chimeric peptide with the ability to bind and incorporate stoichiometric amounts of ATP upon coaggregation and formation of amyloid fibrils.

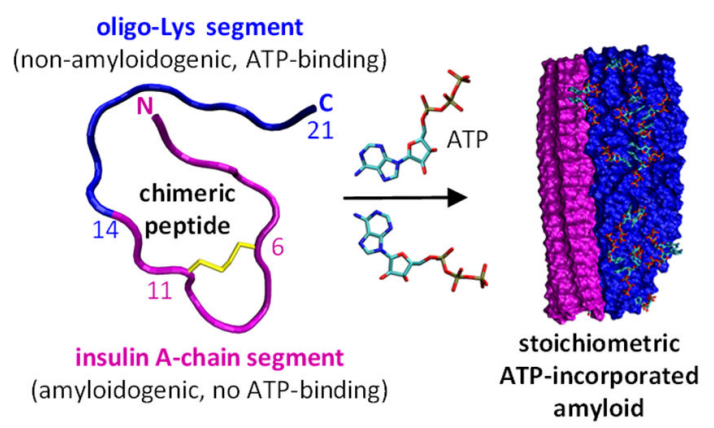

Keywords: · aggregation · amyloid · ATP $\cdot$ fibrils $\cdot$ self-assembly 